This paper presents the evolution of the laws, norms and programs developed in Mexico for the promotion of welfare in the workplace. In Mexico since the 1990s, the obligation to monitor psychosocial risks at work was included in labour legislation, but without definitions of companies' responsibilities and a lack of competencies of the labour authorities to surveille and enforce it. This situation resulted in an absence of surveillance and prevention of psychosocial risk factors and wellbeing promotion in most work sites. Subsequently, with the labour law update in 2012, this law included the obligation to promote decent work and the prohibition of mobbing and sexual harassment, the federal regulations on occupational safety and health were updated and included the actions to prevent psychosocial risks and as something new for Mexico, the companies' obligation to promote favourable organisational environments and health promotion programs. As a result of these changes, a technical standard for the evaluation of psychosocial risks was issued and the Labour Secretary established a National Program for Emotional Well-being and Human Development to promote workers' wellbeing, this program that has been very successful in its implementation in workplaces. This program follows the ILO SOLVE method, integrating the prevention of psychosocial risks, the improvement of organisational culture of prevention, and activities to promote healthy habits and behaviours.

\section{MANAGING PSYCHOSOCIAL WORKING CONDITIONS}

Robert L Kerr. Ulster University, UK

\subsection{6/oemed-2018-ICOHabstracts. 1688}

This session will explore the recent advancements made in managing psychosocial working conditions within Ireland, the UK and Sweden. The presentations will cover: the benefits of adopting a 'positive' approach to psychosocial risk management, the feasibility of adopting a single-item measure of psychosocial working conditions, intervention approaches used within Ireland, the UK and Sweden to improve working conditions, and rapid methods for evaluating intervention effectiveness.

${ }^{1} \mathrm{R}$ Kerr, ${ }^{2} \mathrm{P}$ Buckley, ${ }^{3} \mathrm{~J}$ Houdmont, ${ }^{4} \mathrm{R}$ Randall, ${ }^{5}$ Hultgren

${ }^{1}$ Ulster University, UK

${ }^{2}$ Heath and Safety Executive, UK

${ }^{3}$ University of Nottingham, UK

${ }^{4}$ Loughborough University, UK

${ }^{5}$ The Coaching Psychology Unit, University of London, UK,

\section{$1672 a$ MANAGING PSYCHOSOCIAL WORKING CONDITIONS WITHIN IRELAND AND THE UK}

${ }^{1} \mathrm{R}$ Kerr ${ }^{2} \mathrm{P}$ Buckley. ${ }^{1}$ UIster University, UK; ${ }^{2}$ Heath and Safety Executive, UK

10.1136/oemed-2018-ICOHabstracts. 1689

The psychosocial domains identified by the Management Standards are essentially bipolar in nature as they carry both pathogenic and salutogenic potential. Consider, for example, the psychosocial hazard of social support at work, where a multitude of studies have shown that low levels of support may have a negative impact on an individual's health; yet, conversely, high levels may protect and enhance it.
Psychosocial work interventions such as the HSE's Management Standards can move beyond an exclusive focus on risk assessment and reducing the number of employees at the bottom end of the mental health spectrum toward embracing the potential to make a positive contribution to the mental health of the workforce as a whole.

This presentation will explore the recent advancements made in managing psychosocial working conditions within Ireland and the UK. We will describe how the Irish Health and Safety Authority and State Claims Agency have recently embraced a 'positive' approach to psychosocial risk management through the Work Positive ${ }^{\mathrm{CI}}$ online tool. Work Positive ${ }^{\mathrm{CI}}$ assesses workplace stressors, employee psychological wellbeing and critical incident exposure in the workplace and places a focus on developing clear action plans. We will also present some initial research findings lending further support to a balanced approach.

In addition, we describe the results of a population intervention by the UK's Health and Safety Executive (HSE) to decrease the psychosocial risks in the UK population using the Management Standards approach 2004-2010 and discuss current policy and future HSE interventions on work related stress, in schools in Liverpool, NHS Trusts in Scotland and correctional facilities in the North West of England 20162020 .

\section{2b VALIDATION OF A SINGLE-ITEM MEASURE OF JOB STRESSFULNESS}

${ }^{1} \mathrm{~J}$ Houdmont ${ }^{2} \mathrm{R}$ Randall. ${ }^{1}$ University of Nottingham, UK; ${ }^{2}$ Loughborough University, UK

\subsection{6/oemed-2018-ICOHabstracts. 1690}

Single-item measures have become popular for the assessment of job stressfulness among researchers and practitioners keen to limit assessment burden and interruption to work activities, while maximising response rates in psychosocial risk assessment. A typical single-item measure invites respondents to indicate the degree to which they find their job stressful on a 5 -point scale of
1. not at all,
2. mildly,
3. moderately,
4. very, and
5. extremely,

with responses of 4 or 5 being indicative of high job stressfulness. Despite the popularity of this measure with practitioners there remains a paucity of validation evidence. This validation study aimed to determine the extent to which a response of 4 or 5 was associated with self-reported cases of common mental disorder (CMD). Police officers from two English county forces completed a self-report questionnaire to report their overall job stressfulness, psychological distress, and burnout. We established associations between high job stressfulness and CMD cases using binary logistic regression to generate odds ratios (ORs) and $95 \%$ confidence intervals (CIs). Scores on the three measurement instruments were obtained from 1226 officers (48\% response rate). Forty per cent of respondents reported high job stressfulness, 52\% high psychological distress (PD), 51\% high emotional exhaustion (EE), 47\% high depersonalisation (DP), and 68\% low personal accomplishment (PA). The ORs for PD (OR 8.84, CI: 
6.73 to 11.60 ), EE (OR 11.81, CI: 8.90 to 15.66 ), DP (OR 2.88, CI: 2.27 to 3.64 ), and PA (OR 1.88 , CI: 1.46 to 2.43 ) were significantly elevated for those reporting high job stressfulness. These findings indicate that responses to the single-item measure of job stressfulness, with a cut off drawn at $\geq 4$, differentiated between cases and non-cases across wellbeing indices. A single-item measure of job stressfulness might represent an efficient first pass psychosocial risk assessment for the identification of areas that warrant in-depth assessment and targeted risk reduction activities.

\section{C RAPID PROCESS EVALUATION OF ORGANISATIONAL- LEVEL PSYCHOSOCIAL INTERVENTIONS}

${ }^{1} \mathrm{R}$ Randall ${ }^{2 \mathrm{~J}}$ Houdmont ${ }^{3} \mathrm{~K}$ Nielsen. ${ }^{1}$ Loughborough University, UK; ${ }^{2}$ University of Nottingham, UK; ${ }^{3}$ University of Sheffield, UK

\subsection{6/oemed-2018-ICOHabstracts. 1691}

Many researchers and policy makers have recommended that organizational-level interventions (such as participatory changes to job design) should be used as a first resort when tackling work-related stress. However, there is limited evidence that points to the effectiveness of these interventions. In this presentation we will argue that these interventions need to be better monitored, managed and modified in order to fit workers' diverse and fluctuating needs and circumstances. Findings from a variety of organizational-level intervention studies point to the need for rapid intervention process evaluation. There is growing evidence that: participatory organisational change processes involve demands that may not be universally welcomed; workers' individual differences mean that there is heterogeneity in change impacts; the working conditions targeted for intervention are not equally problematical for all employees; workers' evaluations of intervention activities can significantly vary between individuals and across time; and intervention activities may not always fit neatly into pre-existing hierarchical organisational structures. These problems mean that organizational-level intervention processes need to be more responsive to workers' heterogeneous values, preferences, needs, experiences, competencies, perceived work demands and work contexts. We will describe how intervention process evaluation data can be quickly and frequently gathered to identify the contextual factors and individual differences that support (or inhibit) intervention exposure and outcomes. This will include a presentation of prototype measures and a discussion of practical examples of the ways in which process evaluation data can be used to make intervention activities better fit employees' needs and circumstances.

\section{2d TEAM COACHING AS AN INTERVENTION IN THE WORKPLACE PSYCHOSOCIAL RISK ASSESSMENT PROCESS}

${ }^{1} \mathrm{U}$ Hultgren, ${ }^{2} \mathrm{R}$ Berglund, ${ }^{2} \mathrm{~T}$ Backström. ${ }^{1}$ The Coaching Psychology Unit, University of London, UK; ${ }^{2}$ Mälardalen University, Eskilstuna, Sweden

10.1136/oemed-2018-ICOHabstracts. 1692

Introduction This paper presents an ongoing experimental longitudinal study investigating whether solution focused cognitive behavioural team coaching (SF-CBTC) has a contributory role within systematic workplace psychosocial risk assessments (PS$\mathrm{RA})$ to increase wellbeing. Incorporating SF-CBTC into the organisations already existing processes and roles, could potentially offer a more proactive approach. The inclusion of a structured team coaching method could potentially strengthen step 3-5, of the Health and Safety Executives Management Standards risk assessment process or approach (Cousin, et al., 2004, Kerr, et al., 2014). Adding additional structure/process/ method, for managers team coaching, could assist in orienting the dialogue towards a practice-based solution focused mind set rather than a problem orientation focus when finding solutions to issues identified in the PS-RA. The research is funded by AFA Insurance, an organisation owned by Sweden's labour market parties.

Method The research is being performed in two global technology and manufacturing companies in Sweden. The research groups involves 150 participants consisting of 20 leaders and their teams and a control group of 150 employees, measured at three time points. The design involves 5 steps:

- Education in:

a. Psychosocial safety and

b. 'Leader as a coach' including a SF-CBTC method, PRACTICE (Palmer, 2011; Hultgren, et al., 2013).

- Assessment: Work Positive Profile (Cousins, et al., 2004), adapted research version, measuring:

a. Well-being,

b. Stress factors,

c. Psychosocial safety,

d. Performance and

e. Climate for innovation.

- Root cause analysis

- SF-CBTC coaching intervention

- Peer coaching.

Results The results are expected to show if SF-CBTC could be a valid method to further investigate when using PS-RA for improving factors in the psychosocial work environment and well-being in teams. Secondary outcomes may also show if coaching methods could affect or create a positive coaching culture that could 'live on' in the team, facilitating communication, learning and solutions focus, also after PS-RA intervention is finalised.

\section{OFF JOB EXPERIENCES, HEALTH AND WELL-BEING}

${ }^{1}$ Akihito Shimazu*, ${ }^{2}$ Masaya Takahashi*. 'Kitasato University, Sagamihara, Japan; ${ }^{2}$ National Institute of Occupational Safety and Health, Kawasaki, Japan

\subsection{6/oemed-2018-ICOHabstracts. 1693}

This session aims to discuss about off-job experiences, health, and well-being by focusing on daily rest periods and leisure crafting

Dr. Masaya Takahashi ${ }^{1}$, Dr. Jessica de Bloom ${ }^{2}$, Dr. Sara Arphorn $^{3}$

${ }^{1}$ National Institute of Occupational Safety and Health, Kawasaki, Japan

${ }^{2}$ University of Tampere, Tampere, Finland

${ }^{3}$ Mahidol University, Bangkok, Thailand 